\title{
Die verdeckte (submuköse) Gaumenspalte
}

\section{Patienteninformation}

Die Häufigkeit von Lippen- und Gaumenspalten liegt bei etwa 1:2000 Geburten [1], dabei hat die submuköse Gaumenspalte (SMGS) einen Anteil von 5-10\%. Sie ist durch die in der Mittellinie unverbundene und somit nicht funktionsfähige Weichgaumenmuskulatur gekennzeichnet. Die isolierte Spaltung des Zäpfchens hingegen ist für die Weichgaumenfunktion funktionell bedeutungslos. Bagatin [2] fand unter 9720 6-13-jährigen Schülern 5 Kinder mit einer SMGS, während sich ein isoliert gespaltenes Zäpfchen ohne gleichzeitige Gaumenspalte 232-mal fand.

Zu den 3 typischen Symptomen gehören

- das gespaltene Zäpfchen (eine verdeckte Gaumenspalte kann aber auch bei nicht gespaltenem Zäpfchen vorliegen),

- die tastbare muskuläre Spaltbildung am weichen Gaumen und

- das nicht vorhandene knöcherne hintere Ende des Nasenbeins mit stattdessen tastbarer V-förmige Kerbe am Hinterrand des harten Gaumens [3].

Bei breiter SMGS kann die Schleimhaut über dem muskulären Defekt in der Mittellinie direkt aufeinander liegen und so den Eindruck einer transparenten Zone hervorrufen ( $\bullet$ Abb. 1).

\section{Klinische Symptome \\ $\nabla$}

Durch die in der Mittellinie nicht vereinigten Muskeln des Weichgaumens kommt

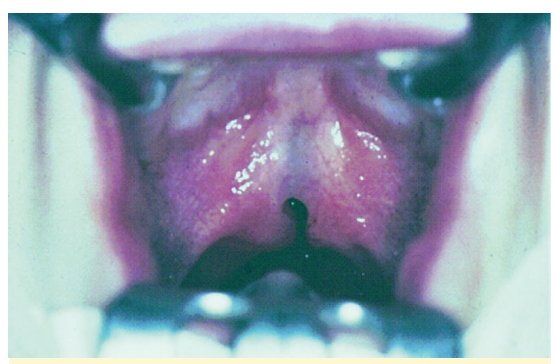

Abb. 1 Man sieht das gespaltene Zäpfchen in Gaumenmitte und die durch die intakte Schleimhaut durchschimmernde Spaltbildung der Weichgaumenmuskulatur (aus [4]). es zu einer unzureichenden Hebung des weichen Gaumens. Symptome einer Gaumenschwäche sind:

- Austreten von Flüssigkeiten beim Trinken oder Erbrechen durch die Nase,

> offenes Näseln,

- chronische Mittelohrbelüftungsstörung mit Paukenerguss und Hörminderung.

Häufig verstärkt sich das offene Näseln nach HNO-ärztlichen Eingriffen wie der Entfernung der Rachen- oder Gaumenmandeln, da sich dadurch die Durchstromöffnung zur Nase vergrößert.

Drei Studien der letzten 20 Jahren [5-7] belegen, dass viele Kinder mit SMGS bis heute nicht rechtzeitig behandelt werden (das mittlere Diagnosealter liegt nach wie vor bei knapp 5 Jahren). Der operative Gaumenverschluss sollte zwischen dem 12 . und 18. Lebensmonat erfolgen $[8,9]$, denn früh versorgte Kinder haben eine bessere Hörfähigkeit und somit auch bessere Voraussetzungen für den Spracherwerb [10].

\section{Therapie der verdeckten Gaumen- spalte (Gaumenspaltplastik) $\nabla$}

Bei der SMGS wird die Gaumenspaltplastik zur Vereinigung der Muskulatur, der Beseitigung des gespaltenen Zäpfchens und der Verlängerung des Gaumens nach hinten (bis $\mathrm{zu} 1 \mathrm{~cm}$ ) durchgeführt. Der zwar nicht gespaltene, aber von Geburt an zu kurze Gaumen hat als sog. „Minimalform“ einer SMGS funktionell dieselben Auswirkungen. Dabei wird der weiche Gaumen ebenfalls operativ gestrafft und nach hinten verlängert, um so einen vollständigen Abschluss zwischen Rachen und Gaumen zur Nase hin zu erreichen [7-9].

\section{Wann empfiehlt man eine Velopharyngoplastik? \\ $\nabla$}

Wenn eine logopädische Behandlung nach dem Gaumenspaltverschluss nicht ausreicht, um das noch bestehende offene Nä- seln zu beseitigen, wird der Rachen zusätzlich plastisch chirurgisch verengt (Velopharyngoplastik). So kann der weiche Gaumen sehr weit nach oben verlängert werden. Leider führt dieses teilweise Vernähen der Weichgaumenmuskulatur häufig zu einer Verstärkung von Ohrproblemen, so dass dieser Eingriff sorgfältig überlegt werden muss. Bei stark vergrößerten Gaumenmandeln können die Länge und Beweglichkeit des Weichgaumens meist nicht zuverlässig beurteilt werden. Vor allem bei gleichzeitig gespaltenem Zäpfchen muss bedacht werden, dass es nach der Gaumenmandelentfernung zu einer Gaumenschwäche kommen kann, welche ggf. einer weitergehenden logopädischen oder operativen Behandlung bedarf.

\section{Fazit}

Die SMGS und der angeborene zu kurze Gaumen machen sich klinisch durch ein offenes Näseln, den Austritt von Flüssigkeiten beim Trinken aus der Nase und chronische Paukenergüsse bemerkbar. Die Therapie der Wahl ist die Gaumenspaltplastik im 12.-18. Lebensmonat. Symptome können sich nach HNO-ärztlichen Eingriffen an den Gaumen- und der Rachenmandel verstärken. Ein gemeinsames Vorgehen verschiedener Fachdisziplinen ist in unklaren Fällen unerlässlich. Gegebenenfalls kann der HNO-ärztliche Eingriff gemeinsam mit dem Gaumenspaltverschluss erfolgen und dem Kind so unnötige Komplikationen und eine zusätzliche Narkose ersparen.

\section{Sibylle Brosch, Ulm}

\section{Literatur}

Die Literatur finden Sie unter www.thiemeconnect.de/ejournals/ssg

überreicht durch Thieme und 


\section{Literatur}

1 Calzolari E, Bianchi F, Rubini M et al. Epidemiology of cleft palate in Europe: Implications for genetic research. Cleft Palate Craniofac J 2004; 42: 244-249

2 Bagatin M. Submucous cleft palate. J Max Fac Surg 1985; 13: 37-38

3 Calnan LS. Submucous cleft palate. Brit J Plast Surg 1954; 6: 264-282

4 Brosch S, Haase S: Näseln und velopharyngeale Insuffizienz bei Adenotomie und Tonsillektomie. Laryngo Rhino Otol 2007; 86: 665-675

5 Brosch S, Kalehne P, Haase S et al. Die submuköse Gaumenspalte in der Praxis. Monatsschr Kinderheilkd 1998; 146: 12021205

6 Johannsen HS, Haase S. Nasal speech with special reference to a submucous cleft plate. Laryngo Rhino Otol 1988; 67: 599-602

7 Reiter R, Haase S, Brosch S. Die submuköse Gaumenspalte - eine oft verkannte Fehlbildung. J Laryngo Rhino Otol 2010; 89: 29-33

8 Porterfield HW, Trabue JC. Submucous cleft palate. Plast Reconstr Surg 1965; 35: 45-50

9 Rohrich RJ, Leve EJ, Byrd HS et al. Optimal timing of cleft palate closure. Plast Reconstr Surg 2000; 106: 413-423

10 Brosch S, Flaig S, Bacher M et al. Früher Gaumenspaltverschluss und Tübinger Atmungsgaumenplatte - mögliche Einflüsse auf die Tubenfunktion bei der Pierre Robin Sequenz. HNO 2006; 54: 756-760 